\title{
FAST ROBUST DESIGN OPTIMISATION METHODS APPLIED TO SHIP HYDRODYNAMICS
}

\author{
MARINE 2011 \\ P. GEREMIA*, T. SCHUMACHER $\uparrow$ AND E. DE VILLIERS $\dagger$ \\ ${ }^{*}$ Engys $\mathrm{Srl}$ \\ Via del Pilone 3, 34126 Trieste, Italy \\ e-mail: p.geremia@engys.eu, web page: http://www.engys.it \\ ${ }^{\dagger}$ Engys Ltd \\ 65 Dalmeny Avenue, London, SW16 4RR, United Kingdom \\ Email: \{t.schumacher,e.devilliers\}@engys.eu - Web page: http://www.engys.eu
}

Keywords: Ship hydrodynamics, design optimisation, uncertainty quantification, OPENFOAM, Response Surface Methodology.

Summary. This paper describes the benefits of fast multi-objective design optimisation methods applied to ship hydrodynamics and coupled to Computational Fluid Dynamics models.

\section{INTRODUCTION}

Nowadays optimisation of ship hydrodynamics represents one of the most challenging design tasks due to the complexity of the engineering problem considered and the inherent multi-objective nature of the problem. Therefore, achieving an optimal solution for a given range of design parameters in the shortest time possible is becoming more and more important. In this regard, usage of the Open Source Computational Fluid Dynamics (CFD) software OPENFOAM ${ }^{\circledR 1}$ to perform flow simulations of hull hydrodynamics represents a validated and efficient method applied to ship design. Furthermore, working with parametric CFD models coupled with design optimisation tools like DAKOTA ${ }^{2}$ represents a further significant breakthrough in process design to perform geometrical shape optimisation. Optimisation tools work like a software "robot" which performs automatic design evaluation by (i) creating new models having updated values of design parameters, (ii) running the model simulation and (iii) extracting the response functions for performance assessment. For this purpose, multi-objective design optimisation algorithms drive the automated run as mentioned above to iterate and create new designs until the optimal solution is found. The potential high number of design parameters combinations to be evaluated make the optimisation process a challenging task and the selection of the best optimisation approach a key factor. In this context, highly non linear problems like ship hydrodynamics can be addressed by using advanced multi-objective design optimisation techniques, ranging from the well known genetic algorithms ${ }^{3}$ to the most advanced Response Surface Methodology (RSM ${ }^{4}$. During the last decade, these techniques have been proved to be robust and efficient when applied to complex non linear engineering applications such as CFD modelling. An additional 
requirement when it comes to perform ship hydrodynamics optimisation is the stability and robustness of the optimal solution found for a given range of operating conditions. Application of uncertainty quantification methods plays an important role in case of nondeterministic conditions, thus allowing the designer to look for the optimal solution in terms of performance stability.

The ship model considered for this study is the US Navy Combatant DTMB 5415, a prototype model conceived in 1980 showed in Figure 1.

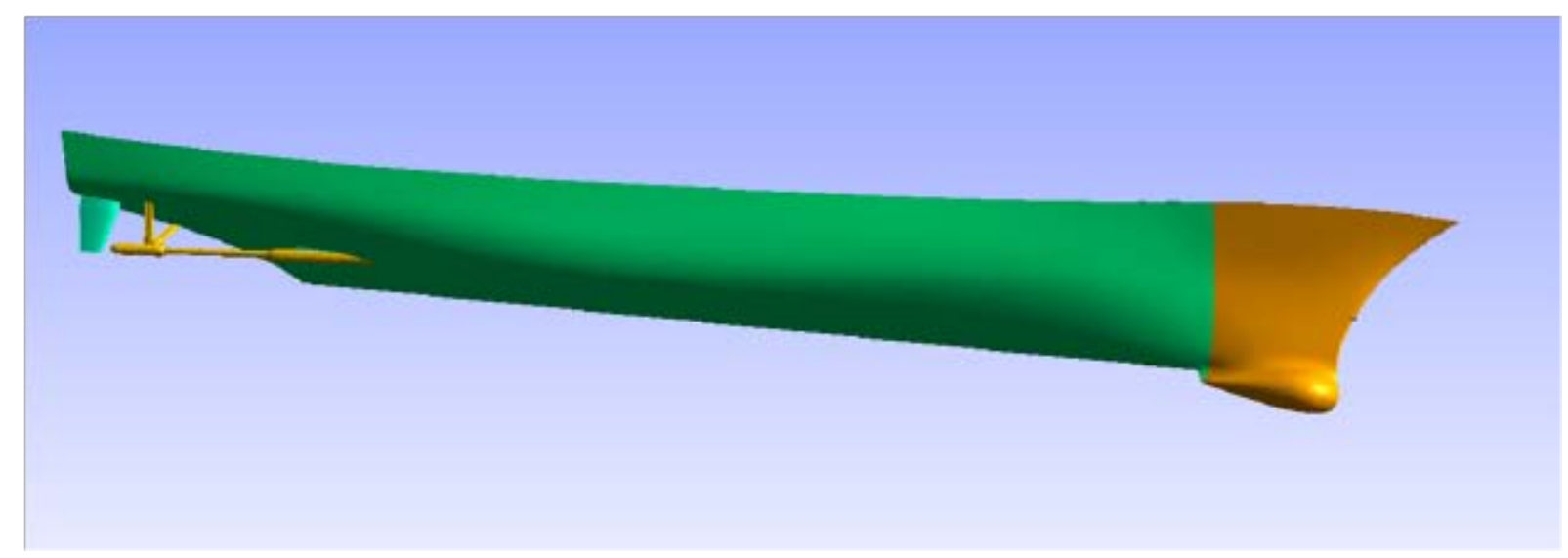

Figure 1: US Navy Combatant DTMB 5415 CAD model

Two open-water propellers whose shaft is supported by stern tubes and brackets are located in the transom stern of the hull. In this paper two different CFD application cases related to ship hydrodynamics were considered, and different fast optimisation approaches were examined in order to highlight the efficiency of the solution proposed applied to the ship design. The first application case is focused on the search of the optimal design of the rudder geometrical shape in terms of drag and lift performance for a given range of variability of operating conditions, including angle of attack and speed. Uncertainty quantification methods were used for this purpose and an efficient fast robust design approach was employed in order to minimise the number of iterations required to find the optimal solution.

The second application case refers to the design optimisation of the bare-hull geometrical model without any appendages considered. The objectives of the study are the minimisation of the ship forward resistance and maximisation of the ship displacement by modifying the geometrical shape of the bow. In this case, a different fast optimisation approach based on RSM techniques was applied, thus considerably reducing the computational time required by the optimisation run.

\section{UNCERTAINTY QUANTIFICATION METHODS}

Uncertainty quantification (UQ) represents a collection of nondeterministic mathematical methods which ideally apply to those engineering problems described by design parameters which are subject to random or irreducible uncertainty. In such cases, design parameters are described by aleatory variables defined via probabilistic methods, when the value of one or more design parameters is known only with some tolerance. 
In UQ the designer looks for the robustness of the solution, in other words for the system capability to be less sensitive to the variation of the design input parameters. Typical applications where UQ techniques perform well are design optimisation of rudder and other appendages for different speeds, angle of attack and other operating conditions. For this purpose, different methods are available in DAKOTA, including Montecarlo techniques, Latin-hypercube Sampling and Polynomial Chaos Expansion (PCE) ${ }^{5}$. All of these methods are based on algorithms which can generate sample points for a numerical approximation of probabilistic distribution applied on top of the nondeterministic design parameters considered. The idea is to map such probabilistic distribution from the design parameters space to the response functions space and check how these distributions propagate. The designer is therefore looking for an optimal solution defined as the one having the lower deviation for a given perturbation applied on the design parameters.

Montecarlo is a sampling technique which spreads the samples over the design space by projecting random points following a specific probabilistic distribution. It can be demonstrated that the number of samples required to estimate the statistical moments (mean and standard deviation) is such that convergence error is proportional to $1 / \operatorname{sqrt}\left(N_{\text {samples }}\right)^{5}$. This means that 100 samples are required to reduce by a factor of 10 the approximation error to compute mean and standard deviation.

Latin Hypercube Sampling is a stratified sampling technique which divides the range of each nondeterministic variable in $N_{\text {samples }}$ segments, all with equal probability, and then selects a random point from each of these segments. The convergence error in this case is proportional to $1 /$ Nsamples, which represents an improvement with respect to the Montecarlo algorithm.

Another valid alternative which can be less computational demanding than the sampling techniques mentioned above is the Polynomial Chaos Expansion (PCE), which allows an exponential convergence by representing a stochastic process as a polynomial expansion in aleatory variables. PCE works like a response surface which maps stochastic input variables to stochastic outputs in order to evaluate statistical moments, namely mean and variance. Expansion methods include the Wiener-Askey scheme ${ }^{5}$, which is based on multivariate orthogonal polynomials to approximate output responses as a weighted function of the probability density of the stochastic inputs. In this way, outputs statistical moments can be expressed as follows:

$$
R=\sum_{j=0}^{P} \alpha_{j} \psi_{j}(\xi)
$$

where $R$ represent the output response, $P$ the expansion order, alpha the $j$-th coefficient, $\psi$ the $j$-th orthogonal polynomial of the stochastic input $\xi$. Thanks to the orthogonal properties of such polynomials it is possible to estimate analytic moments in a more suitable form.

The mean $(\mu)$ can be estimated as follows:

$$
\mu_{R}=\alpha_{0}
$$

Similarly, standard deviation $(\sigma)$ can be expressed as follows:

$$
\sigma_{R}^{2}=\sum_{j=0}^{P} \alpha_{j}^{2}\left\|\psi_{j}\right\|^{2}
$$


The calculation of such polynomial coefficients can be performed using a spectral projection approach through a tensor-product expansion. Therefore, the total number of samples $N_{\text {samples }}$ required to calculate the statistical moments mean and standard deviation is:

$$
N_{\text {samples }}=\prod_{i=1}^{n}\left(p_{i}+1\right)
$$

where $n$ is the number of random variables and $p_{i}$ is the polynomial order for the $i$-th dimension.

To validate the PCE method against traditional Montecarlo and LHS techniques, a simple case adapted from the reliability-based design based optimisation literature ${ }^{6}$ was considered. As showed in Figure 2, PCE exhibits exponential convergence rate for standard deviation moment proportional to $\exp \left(-N_{\text {samples }}\right)$, thus requiring considerably less sampling points with respect to Montecarlo and LHS sampling.

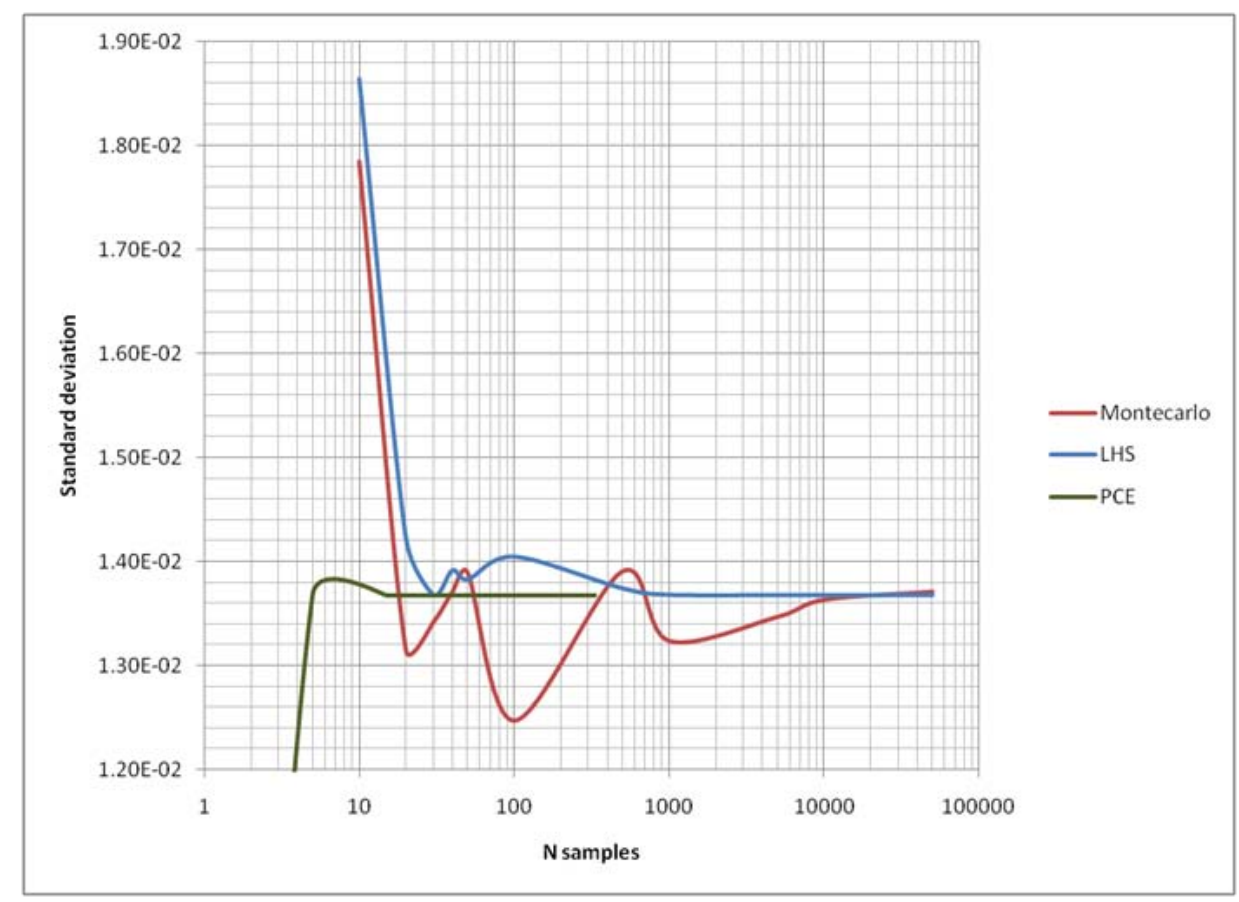

Figure 2: Standard deviation convergence rate using PCE

\section{RESPONSE SURFACE METHODOLOGY}

Response Surface Methodology (RSM) is a collection of techniques often employed to create surrogate models by providing an approximation to an original "high-fidelity" model. In other words, this technique allows the designer to perform a data fit of existing CFD simulation results to create a surrogate model, defined as a function of design variables which can be reused in the optimisation loop, as a way to replace the CFD simulation runs which very often are time consuming and make the total time required for a complete optimisation run prohibitive.

The RSM process consists of (i) preparation of a training set of design points, (ii) evaluation using CFD simulation on these design points and (iii) training of RMS algorithms 
to find the best interpolation function in terms of data fit. Different methods are available in DAKOTA, including linear, quadratic and cubic polynomial models via least-squares methods, Kriging interpolation, artificial Neural-Network, MARS, Radial Basis Function and moving least squares model. Several quality metrics are also available to assess quality and accuracy of the RSM model created based on a training set of design points, including performance measurements like RSME, MSE, SSE, and cross-validation techniques like leave-one-out error, general cross-validation and R-squared value. Validation of the RSM model plays an important role when selecting the most suitable RSM model for each output response, and it also strongly affects the quality of results obtained during the subsequent optimisation run where it will replace the CFD simulation results.

\section{ROBUST DESIGN OPTIMISATION OF THE RUDDER}

The first design application case considered in this work was the optimisation of the geometrical shape of the rudder of the US Navy Combatant DTMB 5415. The aim was to keep a high lift-drag ratio for a range of different operating conditions. More precisely the objective of the study was the minimisation of drag and the maximisation of lift for various speeds and angles of attack.

The optimisation was performed on the 2D hydrofoil cross-section of the rudder and consisted of two stages. In the first stage, both angle of attack and speed were kept fixed at their original values, and then the optimal shape of the hydrofoil for lift and drag was found. In the second stage, the best solutions found in the previous step were assessed by taking into account the variability of the operating conditions (i.e. the angle of attack and speed), and at the end of the run the best stable solution was found.

For this purpose, a NACA0015 profile with angle of attack equal to $8 \mathrm{deg}$ was chosen as baseline cross-section. The original profile was made parametric by the superposition of a third order parametric Bézier curve for both the upper and lower hydrofoil profile, as showed in Figure 3.

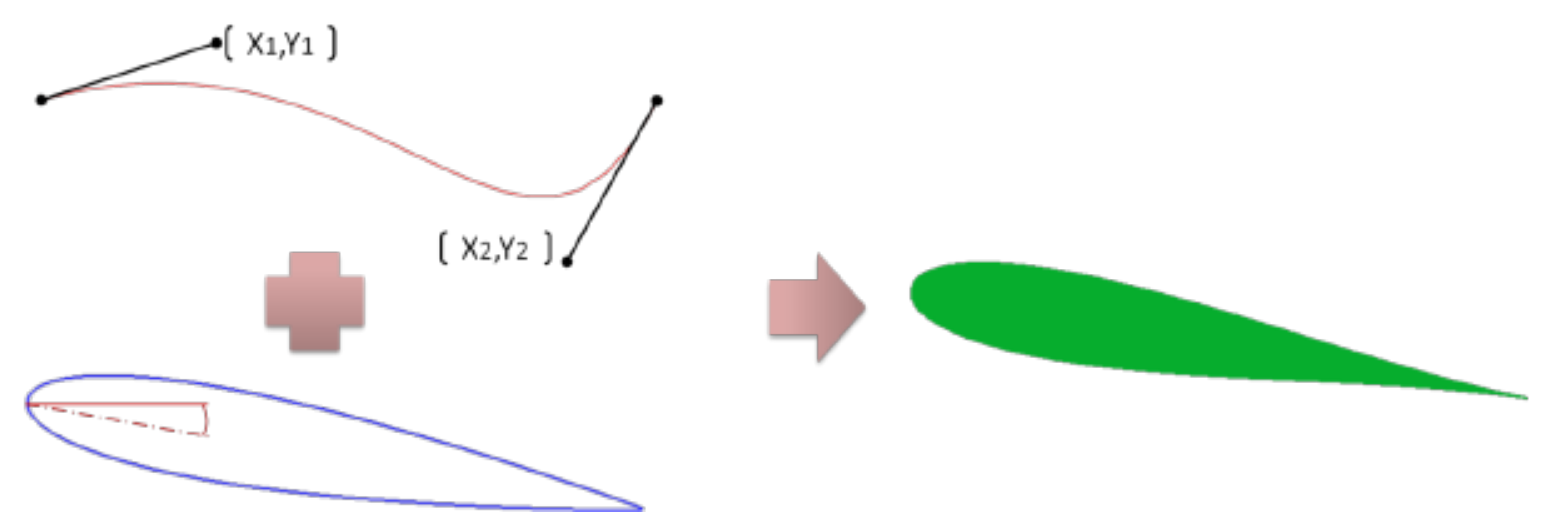

Figure 3: Rudder cross-section parametric shape using Bézier curve method

Four design variables were considered for the definition of the parametric curve, namely the $\mathrm{X}$ and $\mathrm{Y}$ position of two control-points. An automatic hex-dominant polyhedral mesh was created automatically for each design configuration using an enhanced version of the 
snappyHexMesh mesh generator available in OPENFOAM ${ }^{\circledR}$. A subsequent RANS CFD analysis using the $\mathrm{k}-\omega \mathrm{SST}$ turbulence model available in OPENFOAM ${ }^{\circledR}$ was setup. For each design configuration, hydrofoil drag and lift values were computed and stored in a text file. DAKOTA was used as optimisation software and a derivative-free Multi-Objective Genetic Algorithm (MOGA) was applied to perform the initial optimisation phase. Figure 4 highlights the workflow managed by DAKOTA during the optimisation run.

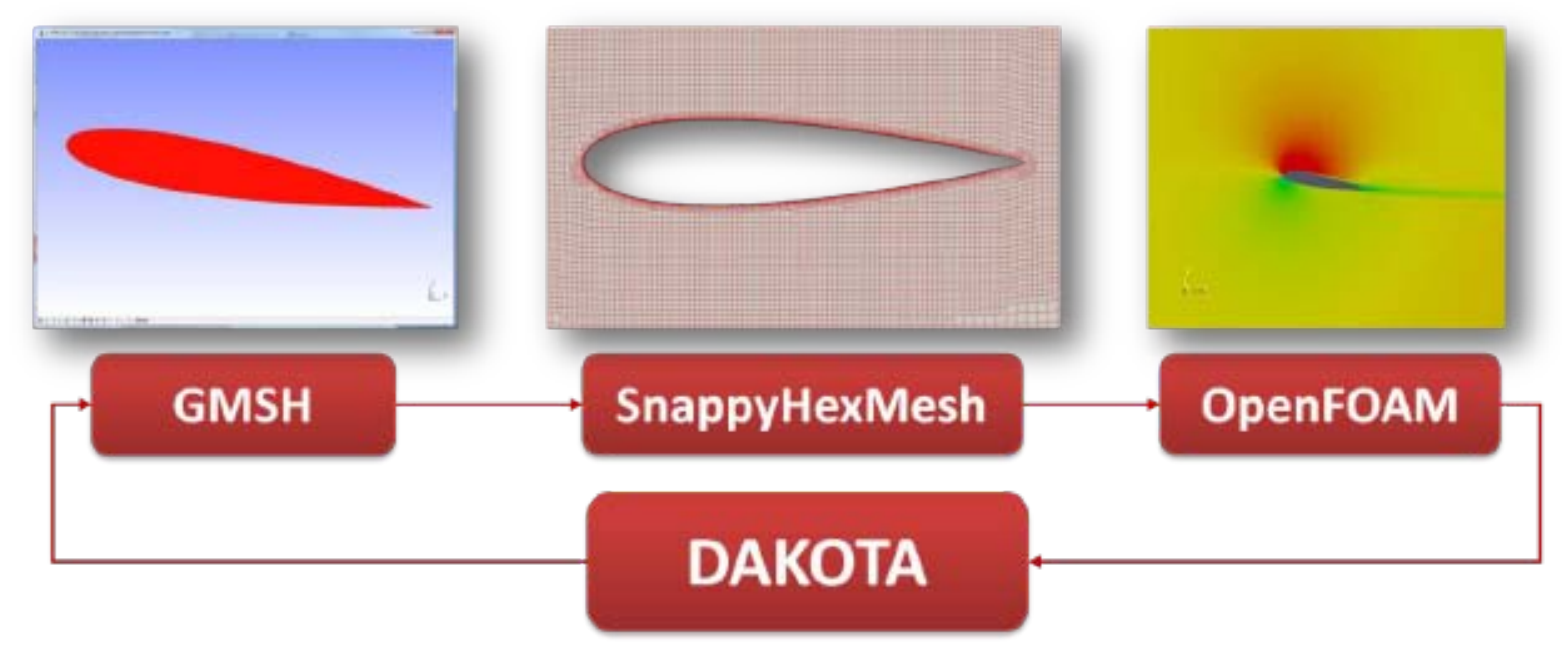

Figure 4: Rudder shape optimisation workflow driven by DAKOTA

The MOGA algorithm was set with a generation size of 20 individuals and asynchronous parallel design evaluations were performed by fully exploiting both DAKOTA and OPENFOAM $^{\circledR}$ license-free scalability. After 35 generations, a set of optimal solutions was found and three different candidate designs were taken for the next step, the first point as best solution for lift, the second for the resistance and the third a compromise solution.

In the next stage of the optimisation phase, the variable operating conditions of the rudder were considered to analyse the response of the system when changing design parameters from nominal operating conditions. In order to take into account the variability of the operating conditions a UQ approach was used, and the stability of the optimal solution for a given range of inlet velocity $U_{i n}$ and angle attack $\alpha$ was evaluated. For this purpose, for each design evaluated a perturbation on $U_{i n}$ and $\alpha$ based on a normal distribution $U_{i n}, \alpha \sim N\left(\mu, \sigma^{2}\right)$ was applied. Consequently, the optimisation problem was re-formulated as follows:

$$
\left\{\begin{array}{c}
M I N\left(\mu_{\text {Resistance }}+3 \sigma_{\text {Resistance }}\right) \\
M A X\left(\mu_{\text {Lift }}-3 \sigma_{\text {Lift }}\right)
\end{array}\right.
$$

A small perturbation was applied on $U_{\text {in }}$ and $\alpha$ using the PCE approach for the three different optimal solutions obtained in the first stage of the optimisation run. A cost-effective design space sampling was performed for accurate prediction of $\mu$ and $\sigma$ of water resistance and lift outputs. For this a 4-th order quadrature PCE sampling was employed, thus leading to 16 design evaluations in total for each design configuration. The previous compromise solution revealed to be a good trade-off both in terms of stability and performance, as showed 
in Table 1.

\begin{tabular}{|c|c|c|c|c|c|c|}
\cline { 2 - 7 } \multicolumn{1}{c|}{} & \multicolumn{3}{c|}{ Resistance } & \multicolumn{3}{c|}{ Lift } \\
\cline { 2 - 7 } \multicolumn{1}{c|}{} & $\mu$ & $\sigma$ & $\mu+3 \sigma$ & $\mu$ & $\sigma$ & $\mu-3 \sigma$ \\
\hline Best Drag & 0.863376 & 0.177527 & 1.395958 & 0.888653 & 0.142788 & 0.460289 \\
\hline Best Lift & 1.034079 & 0.240473 & 1.755499 & 1.056855 & 0.166644 & 0.556922 \\
\hline Compromise & 0.980506 & 0.201355 & 1.584572 & 1.013078 & 0.158264 & 0.538287 \\
\hline
\end{tabular}

Table 1 : Results of robustness study using PCE method

\section{BARE-HULL OPTIMISATION}

The second application case presented in this work is the shape optimisation of the geometry of the bare-hull of the US Navy Combatant DTMB 5415 model. A parametric CFD model of the bare-hull was coupled with the DAKOTA optimisation tool to perform a sensitivity analysis followed by an optimization run to maximise displacement while minimising the forward water resistance.

For this purpose, first the available STL surfaces were loaded into the Open Source surface morphing tool Blender, which allowed different hull geometries to be generated by morphing the geometric shape of the bow. More precisely, two morphing boxes were defined as showed in Figure 5, one for the bow and another one for the bulb. The first box consists of six controls points that can be modified along the $\mathrm{Y}$ and $\mathrm{Z}$ axis; the second box consist of three control points that can be modified along the $\mathrm{Y}$ axis only.
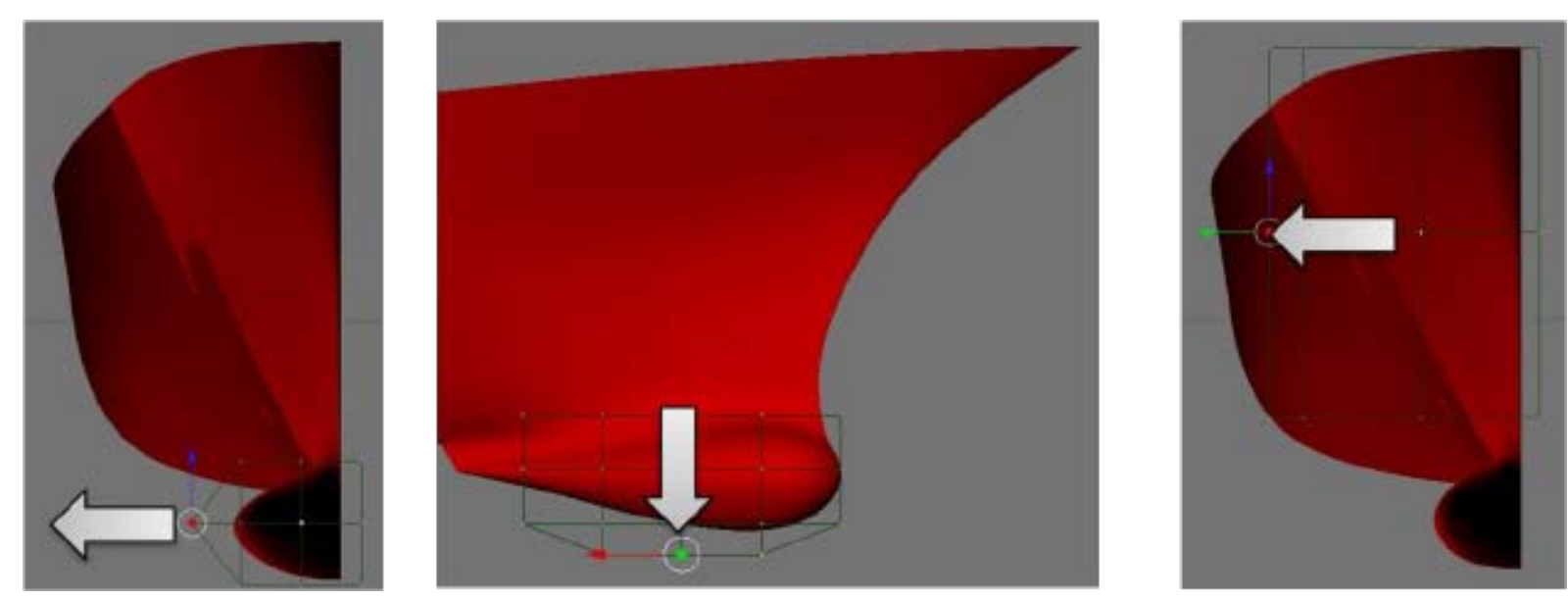

Figure 5: Bare-hull surface morphing parameters for geometry modification

Second, a hex-dominant mesh of 1.5 million cells with five near-wall extrusion layers was created using an enhanced version of the snappyHexMesh tool available in OPENFOAM ${ }^{\circledR}$; the mesh was then employed to define the CFD model showed in Figure 6.

Third, the CFD solutions for displacement and resistance were employed to perform the sensitivity analysis to quantify relationships between design variables and output responses. For this purpose, DAKOTA's Design Of Experiments (DOE) techniques were used to 
efficiently explore the design space and to perform the global sensitivity analysis.

Finally, one hundred sample points were created using a Latin-Hypercube Sampling method and the most significant variables for displacement and resistance output responses were outlined. The 100 initial CFD sample points were used for training a Neural Network RSM to replace the CFD models by surrogate models of both the resistance and displacement output responses. A MOGA optimisation run using response surface models was then carried out and the total time required to run the whole optimisation study was reduced to a few seconds. The layout of the optimal solution was found as a compromise between the resistance and displacement objectives, as showed in Figure 7. The final design selected from the Pareto front was validated afterwards using a high-fidelity CFD analysis.

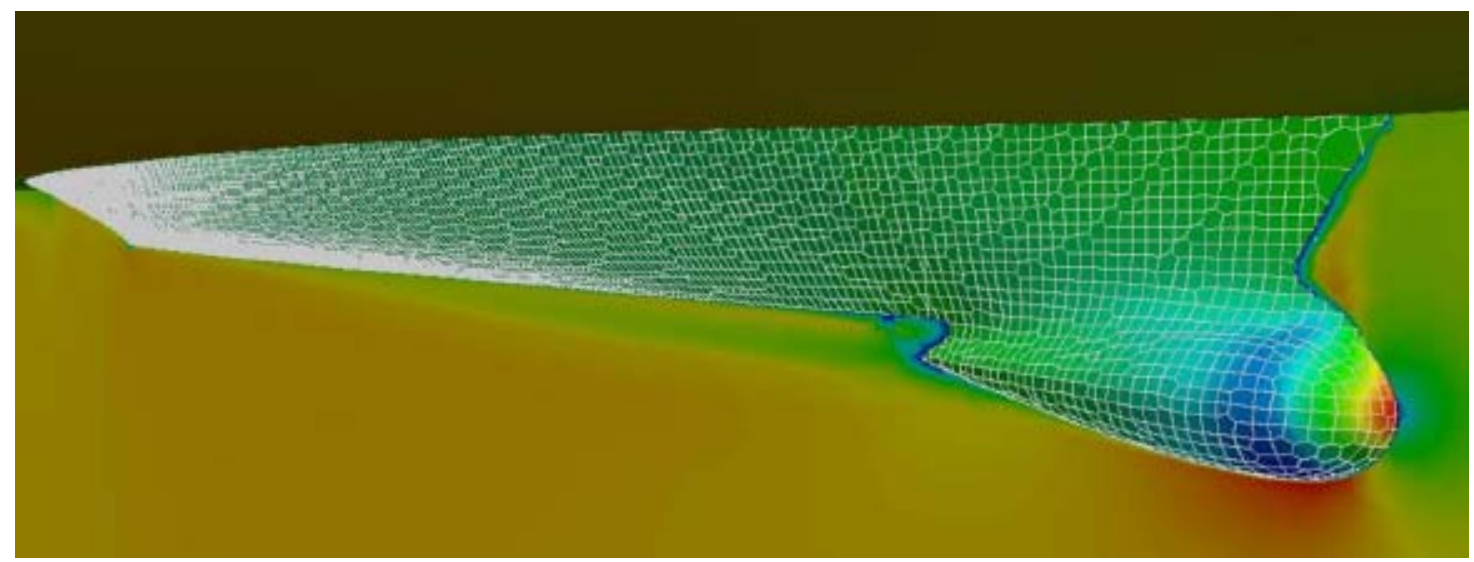

Figure 6: Bare-hull CFD model using OPENFOAM ${ }^{\circledR}$

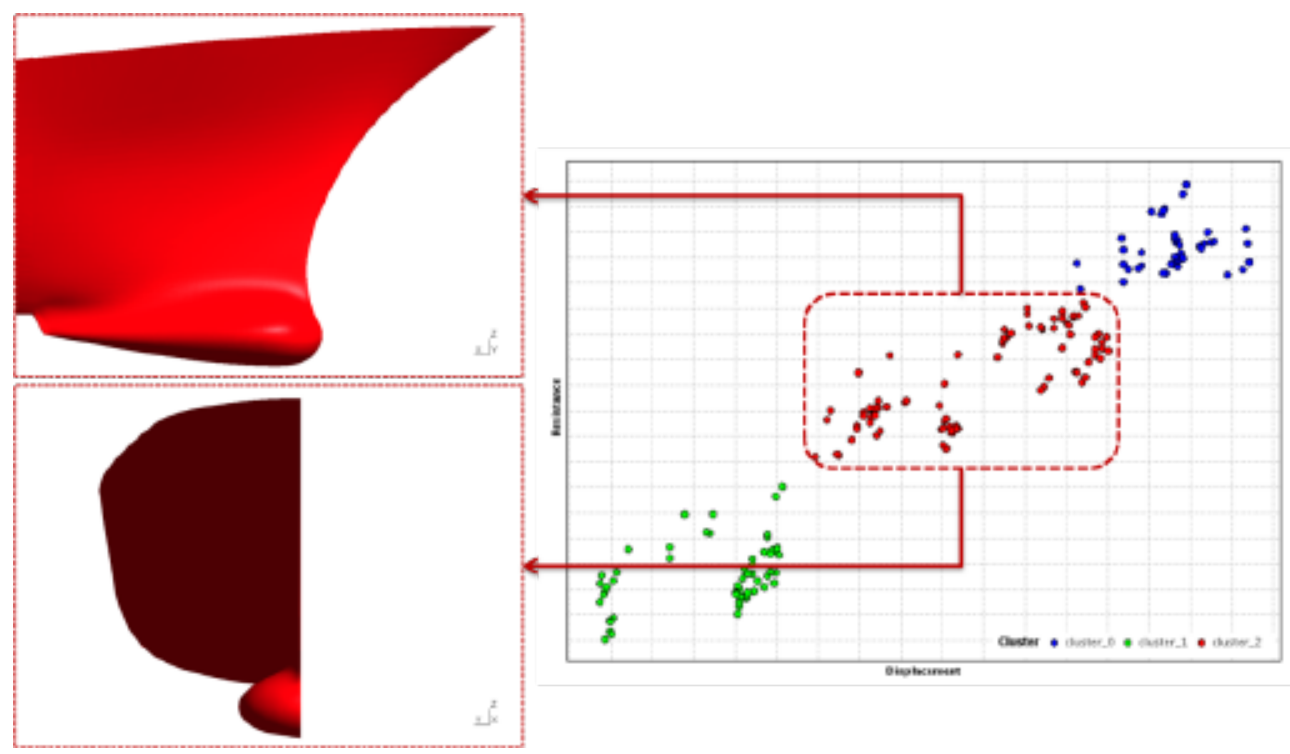

Figure 7: Scatter chart of the objectives for the each design evaluation and optimal solution found 


\section{CONCLUSIONS}

In this work the benefits of Open Source design optimisation methods applied to ship hydrodynamics were highlighted, in particular the advantages of using the CFD platform OPENFOAM $^{\circledR}$ coupled with the multi-objective design optimisation software DAKOTA. Different approaches of geometrical shape parameterisation were evaluated for a couple of test cases, including minimisation of ship forward resistance while maximising displacement, as well as design optimisation of rudder and other appendages for different speed, angle of attack and other operating conditions. Combined application of efficient PCE techniques and parallel computations using OPENFOAM ${ }^{\circledR}$ were exploited to reduce the computational time of the design optimisation runs with a limited number of CFD simulation runs. Cost-effective RSM models were also applied to the model response functions for further speed-up of timeconsuming CFD models without penalising the accuracy of the solution found.

\section{REFERENCES}

[1] OPENFOAM ${ }^{\circledR}$ is a registered trade mark of OpenCFD Limited.

[2] Adams, B.M., Bohnhoff, W.J., Dalbey, K.R., Eddy, J.P., Eldred, M.S., Gay, D.M., Haskell, K., Hough, P.D., and Swiler, L.P., "DAKOTA, A Multilevel Parallel ObjectOriented Framework for Design Optimization, Parameter Estimation, Uncertainty Quantification, and Sensitivity Analysis: Version 5.0 User's Manual" Sandia Technical Report SAND2010-2183, December 2009. Updated December 2010 - Version 5.1 (2010)

[3] N. Marco, S. Lanteri, J.-A. Desideri, J. Périaux, Evolutionary Algorithms in Engineering and Computer Science, Wiley, Chichester, Chapter 22, pp. 445-456 (1999)

[4] Giunta, A. A., "Use of Data Sampling, Surrogate Models, and Numerical Optimization in Engineering Design," paper AIAA-2002-0538 in Proceedings of the 40th AIAA Aerospace Sciences Meeting and Exhibit (2002)

[5] Eldred, M.S., "Design Under Uncertainty Employing Stochastic Expansion Methods", International Journal for Uncertainty Quantification,Vol. 1, No. 2, Feb. 2011, pp. 119$146(2011)$

[6] R. Sues, M. Aminpour, and Y. Shin. "Reliability-based multidisciplinary optimization for aerospace systems", In Proc. 42rd AIAA/ASME/ASCE/AHS/ASC Structures, Structural Dynamics, and Materials Conference, number AIAA-2001-1521, Seattle, WA, April 16$192001(2001)$ 